\title{
Fourier Transformed Infrared Spectroscopy Analysis of Constituents of Lemon Essential Oils from Algeria
}

\author{
Hicham Boughendjioua ${ }^{1, *}$, Samah Djeddi \\ ${ }^{1}$ Department of Natural Sciences, High School Professors Technological Education, Skikda, Algeria \\ ${ }^{2}$ Department of Biology, Faculty of Science, University of Badji Mokhtar, Annaba, Algeria
}

Email address:

boughendjioua.hicham@yahoo.com (H. Boughendjioua)

${ }^{*}$ Corresponding author

\section{To cite this article:}

Hicham Boughendjioua, Samah Djeddi. Fourier Transformed Infrared Spectroscopy Analysis of Constituents of Lemon Essential Oils from Algeria. American Journal of Optics and Photonics. Vol. 5, No. 3, 2017, pp. 30-35. doi: 10.11648/j.ajop.20170503.12

Received: June 9, 2017; Accepted: June 26, 2017; Published: October 30, 2017

\begin{abstract}
Three main tools are used to determine the structures of organic molecules. These tools are infrared (IR) spectroscopy, mass spectrometry (MS) and nuclear magnetic resonance (NMR) spectroscopy. Organic molecules absorb light (infrared, ultraviolet, etc.) at particular wavelengths based on different vibrational modes unique to the specific functional groups and structural features. In the present study, the volatile compounds of Lemon (Citrus limon L.) collected from Collo, Skikda city (Algeria), were detected and identified by Fourier Transformed Infrared Spectroscopy (FTIR) analysis (qualitative identification). FTIR allowed us to identify 10 volatile compounds and indicated than the functional groups of the essential oils are $\mathrm{C}-\mathrm{H}$ (Alkene), $\mathrm{C}-\mathrm{H}$ (aromatic) and $\mathrm{C}=\mathrm{C}$. The obtained results have shown that the essential oils can be fully utilized for pharmacy, cosmetology and industry.
\end{abstract}

Keywords: Lemon, Citrus Limon L., Essential Oil, Fourier Transformed Infrared Spectroscopy (FTIR), Functional Groups

\section{Introduction}

Citrus plants are well-known crops all over the world with potential socio-economic influence. They are well-known for their flavor, nutritional value and medicinal features. The medicinal activities for this genus are attributed to the presence of many medicinally active secondary metabolites such as essential oils [1].

Citrus essential oils have been applied in many products, such as cosmetics, medical formulation, beverages and foods, as flavoring agents as well as for aromatherapy. They are also used for their germicidal, antioxidant and anticarcinogenic properties [2].

Citrus essential oils contain $85-99 \%$ volatile and $1-15 \%$ non-volatile components [3]. The volatile constituents are a mixture of monoterpenes hydrocarbons, sequiterpene hydrocarbons and their oxygenated compounds including [4]: aldehydes (citral), ketones, acids, alcohols (linalool) and esters $[5,6]$. The main volatile compounds presenting in the citrus essential oils are pinene (there are two isomers: alphapinene and betapinene), limonene, citral, myrcene, linalool, á-terpineol (there are five isomers: alpha- terpineol, betaterpineol, gamma- terpineol, delta- terpineol and 4- terpineol) present in different percentages.

Terpineol is usually a mixture of these isomers having alpha-terpineol as major constituent. The chemical structures of the compounds listed above are shown in Figure 1. The major chemical component of the citrus oils is limonene and it varies between $68-98 \%$ in sweet orange, $45-76 \%$ in lemon and $32-45 \%$ in bergamot $[3,7]$.

Most people either use essential oils for their therapeutic effect or for the fragrance alone but it is also interesting to take note of the chemistry, of which the oils are made up from.

Essential oils, like all organic compounds, are made up of hydrocarbon molecules and can further be classified as terpenes, alcohols, esters, aldehydes, ketones and phenols etc.

Every single oil normally has more than a hundred components, but this figure can also run into thousands, depending on the oil in question.

(a). The various organic components found when analyzing essential oils with a chromatograph are: 
(b). Terpene hydrocarbons: Monoterpene hydrocarbons, Sesquiterpenes.

(c). Oxygenated compounds: Phenols, Alcohols.

(d). Monoterpene alcohols.

(e). Sesquiterpene alcohols: Aldehydes, Ketones, Esters, Lactones, Coumarins, Ethers, Oxides [8].

Fourier Transformed Infrared Spectroscopy (FTIR) is based on the absorption of infrared radiation by the analysed material. Through the detection of the characteristic vibrations of the chemical bonds, it makes it possible to carry out the analysis of the chemical functions present in the material [9].

The aim of this work was to clearly describe the functional groups of Lemon essential oils in order to better define the field of investigation of this natural product.

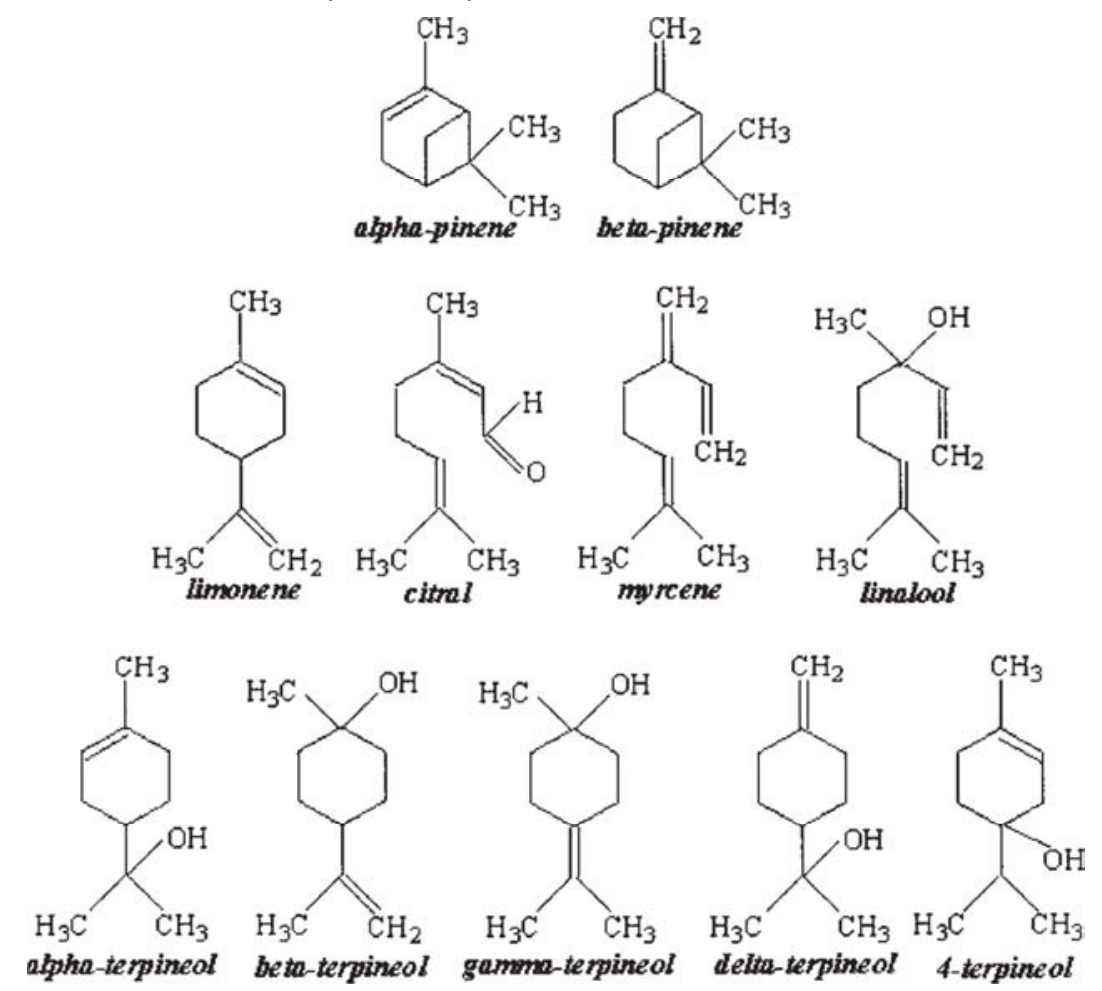

Figure 1. The Chemical Structures of the Main Volatile Compounds Present in the Citrus Essentials Oil.

\section{Material and Methods}

\subsection{Plant Material}

$400 \mathrm{~g}$ of the tested sample was obtained from a private farm located in the region in Collo (Skikda city, North-East of Algeria). Plant harvesting was carried in March 2013. The upper part of the pericarp was harvested from fresh fruit, this choice was justified by the richness of zest in essential oils compared to other parts of the fruit [10].

\subsection{Isolation of the Essential Oil}

Obtaining essential oils was carried out by cold expression (physical process), it's the simplest processes applied only to citrus fruit [11], this extraction does not change the composition of the oil [12]. The product obtained is called gasoline, because it does not undergo any chemical modification $[11,13]$. The obtained essential oil was stocked at $4{ }^{\circ} \mathrm{C}$ until further analyses.

\subsection{FTIR Analysis}

Spectroscopy is based on the study of the interactions between matter and electromagnetic radiation. This radiation consists of a particle beam having an undulating motion. All electromagnetic radiation forms the electromagnetic spectrum. In the spectrum, four regions are discernible: $\mathrm{X}$ rays, ultraviolet (UV), visible and infra-red (IR). The emphasis will be on infrared (IR) spectroscopy because spectrum analysis allows us to follow a reaction process, determine the dosage of a compound, check the purity of a product and identify an unknown. Moreover, it is an inexpensive and easy to use process which makes IR spectroscopy the most widely used spectral method used by chemists. The principle is based on molecular vibrations. The energy emitted as photons can be absorbed by the material causing vibration in the molecules. This vibration changes the angle and the distance between the atoms.

When the molecule returns to its original form, energy will be released as heat. The absorption and release of energy by the molecule will be recorded by the apparatus and translated into a band spectrum. The analysis of this spectrum makes it possible to obtain the necessary information on the material analyzed.

Qualitative information: The wavelengths to which the sample absorbs are characteristic of the chemical groups present in the analyzed material.

Quantitative Information: The intensity of absorption at 
the characteristic wavelength is related to the concentration of the chemical group responsible for absorption.

This method of analysis is simple to implement and not destructive. It allows the analysis of both organic and inorganic materials [9].

FTIR is performed with a PERKIN ELMER (universal ATR Sampling Accessory) apparatus, the operating conditions are as follows: technique: ATR, analysis range: 4000- $600 \mathrm{~cm} \mathrm{-1.} \mathrm{The} \mathrm{results} \mathrm{are} \mathrm{directly} \mathrm{compared} \mathrm{with}$ those of the internal bibliography of the apparatus; 01 . Euclidean, 02. PSU / peak, 03. MIX PSU, 04. Peak Match, 05. PEAK / psu, 06. MIX PEAK. In the present work the Euclidean library has been used. The FTIR analysis was performed at the Regional Police Scientific Laboratory (Constantine, Algeria).

\section{Results and Discussion}

Determination of the Functional Groups Present Using FTIR

Fourier transformed infrared spectroscopy is one of the most widely employed techniques for functional groups identification. Figures 2 to 3 and Table 1 showed the infrared spectra and the characteristic bands observed in Lemon essential oil in the range of 4000-600 cm:

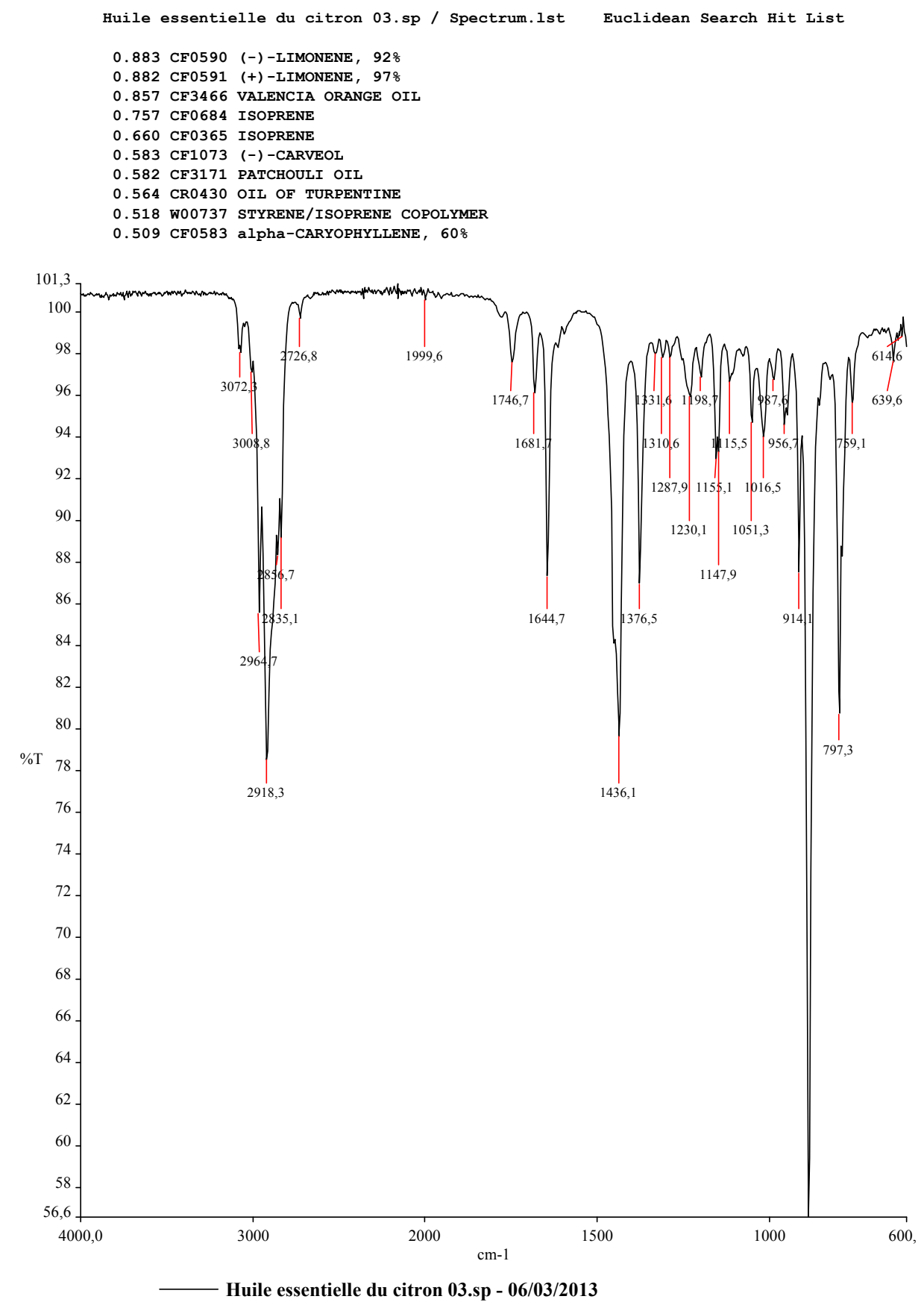

Figure 2. FTIR of Lemon Essential Oil. 


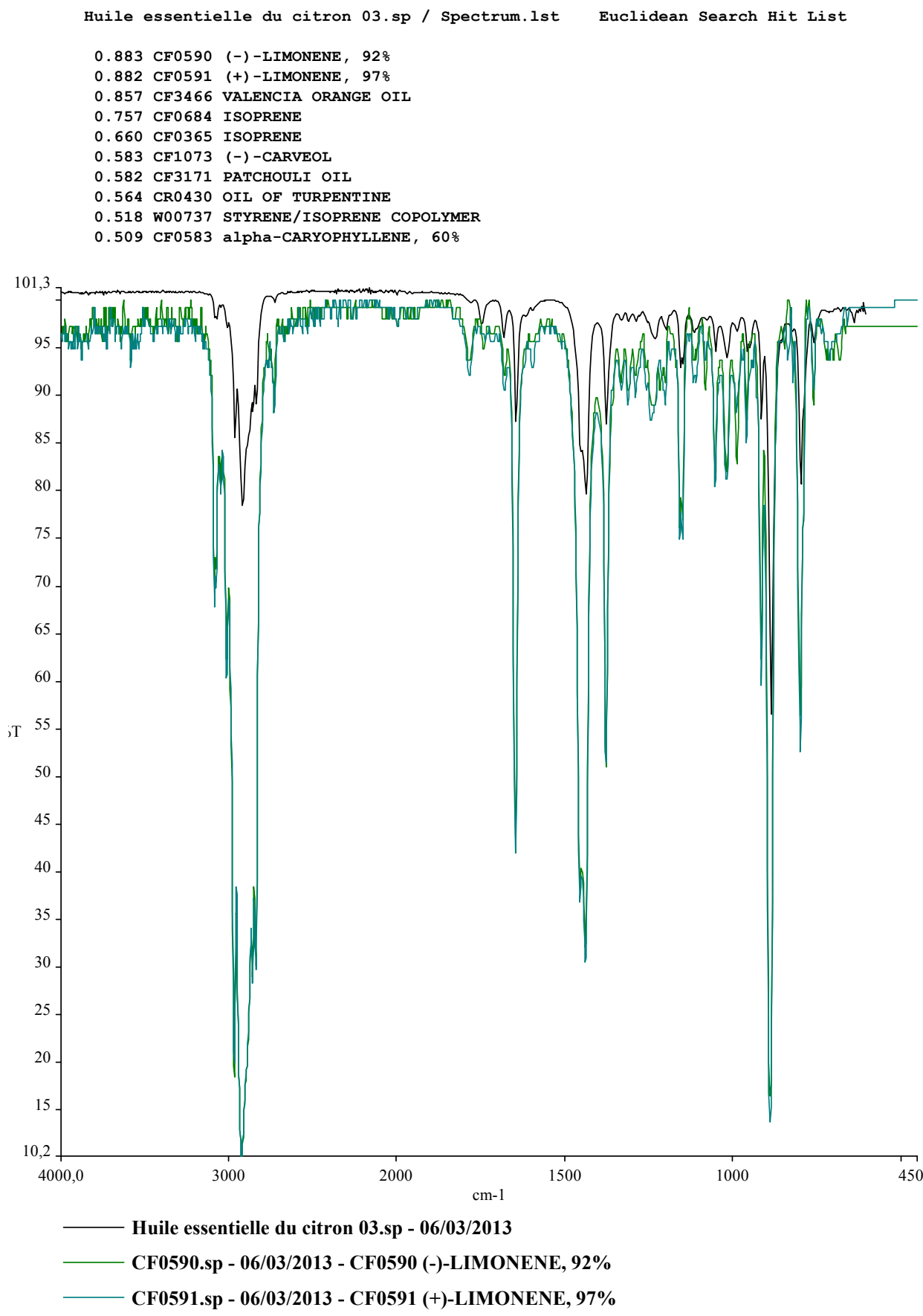

Figure 3. FTIR of Lemon Essential Oil Compared to the Bibliography.

Table 1. Links Present in the Molecule of Limonene.

\begin{tabular}{lll}
\hline Present links in Limonene & Theoretical frequency of the band $\left(\mathbf{c m}^{-1}\right)$ & Presence of the band in the spectrum \\
\hline C-H (Alkene) & $3100-3000$ & Yes \\
C-H (aromatic) & $3150-3050$ & Yes \\
C=C & $1600-1680$ & Yes \\
\hline
\end{tabular}

In an earlier work developed by Boughendjioua and Djeddi (2014) [14], the chemical composition of the essential oil of Citrus limon detected by GC-MS allowed us to identify 53 compounds and indicated that the main compounds constituting the volatile oil were mainly Limonene (61.64\%), $\beta$-pinene (13.85\%) and $\gamma$-terpinene $(9.95 \%)$.

The spectrum of Limonene as well as that of pure essential oils had several similarities (Figure 1 and 2) Unfortunately, no "fingerprint" of the extracted oil coincided with that of pure essential oils or Limonene.

Among the ten compounds revealed by FTRI: (1). (-) limonene, 92\%, (2). (+) - limonene, 97\%, (3). Valencia orange oil, (4). Isoprene, (5). Isoprene, (6). (-) - carveol, (7). Patchouli oil, (8). Oil of turpentine, (9). Styrene / isoprene 
copolymer, (10). Alpha-caryophyllene, 60\%.

On the other hand, the complete and detailed study of a spectrum is an operation rarely practiced in current interpretation because of the complexity of the analysis. It is therefore often limited to the identification of functional groups through the location of the different bands on the spectrum.

The spectrum presents characteristic bands at 1600-1680 $\mathrm{cm}^{-1}$ corresponding to $\mathrm{C}=\mathrm{C}$, the signals which appeared between $3100-3000 \mathrm{~cm}^{-1}$ and $3150-3050 \mathrm{~cm}^{-1}$ are caused by the asymmetrical and symmetrical stretching vibrations of $\mathrm{C}$ $\mathrm{H}$ groups (Figure 4 and 5).

It should be noted that Alkane $\mathrm{C}-\mathrm{H}$ bonds are fairly ubiquitous and therefore usually less useful in determining structure. For C-H Stretch with characteristic absorption $\left(\mathrm{cm}^{-}\right.$ $\left.{ }^{1}\right) ; 3100-3010(\mathrm{~m})$, absorption peaks above $3000 \mathrm{~cm}^{-1}$ are frequently diagnostic of unsaturation [15].

According to Elzey et al., (2016) [16] the FTIR specter of the pure essential oil of Lemon, showing the expected characteristic C-H stretch $(\sim 2900 \mathrm{~cm}-1), \mathrm{C}=\mathrm{O}$ stretch $(\sim 1700$ $\mathrm{cm}-1)$, broad $\mathrm{O}-\mathrm{H}$ stretch $(\sim 3400 \mathrm{~cm}-1)$, and $\mathrm{C}-\mathrm{O}$ stretch ( 1100 cm-1) of terpenoid components. The compositions and constituents of essential oils may vary and highly depend on the geochemistry of the soil where it is cultivated. In general, essential oils are made up of terpenes such as terpineol, cineole, citronellal, and others.

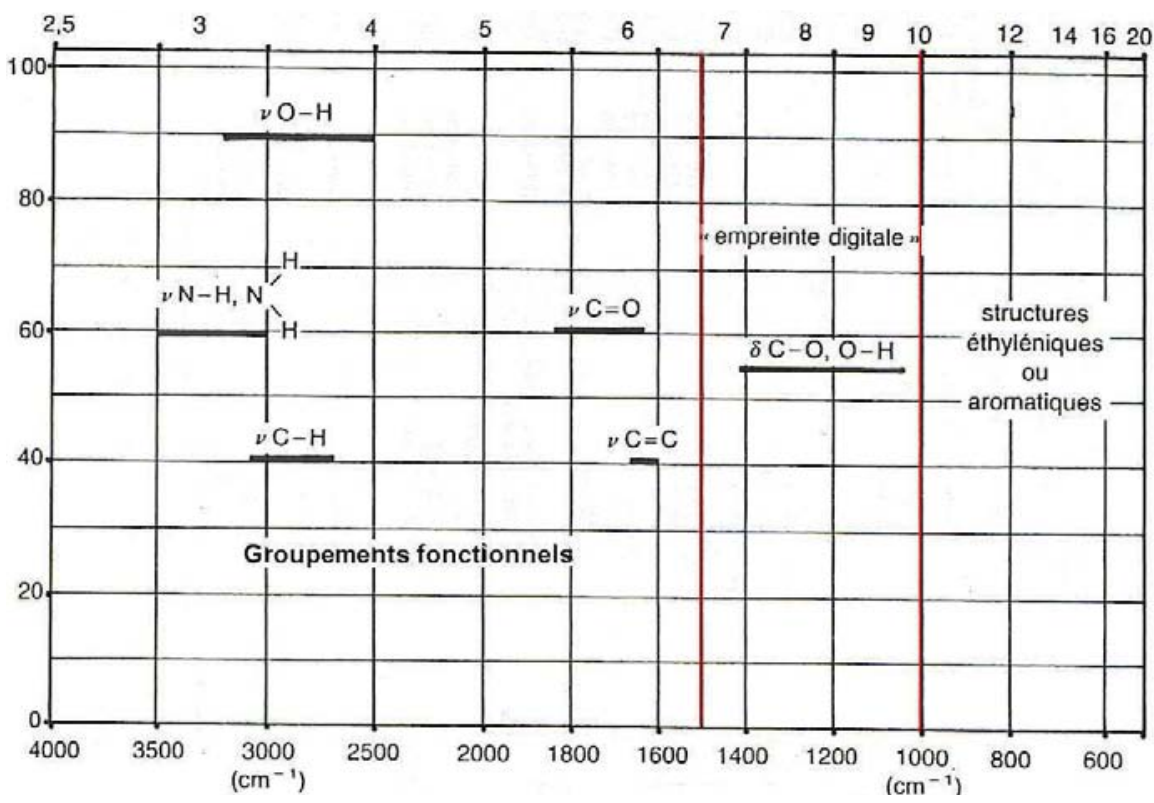

Figure 4. Graphs of IR Spectroscopy.

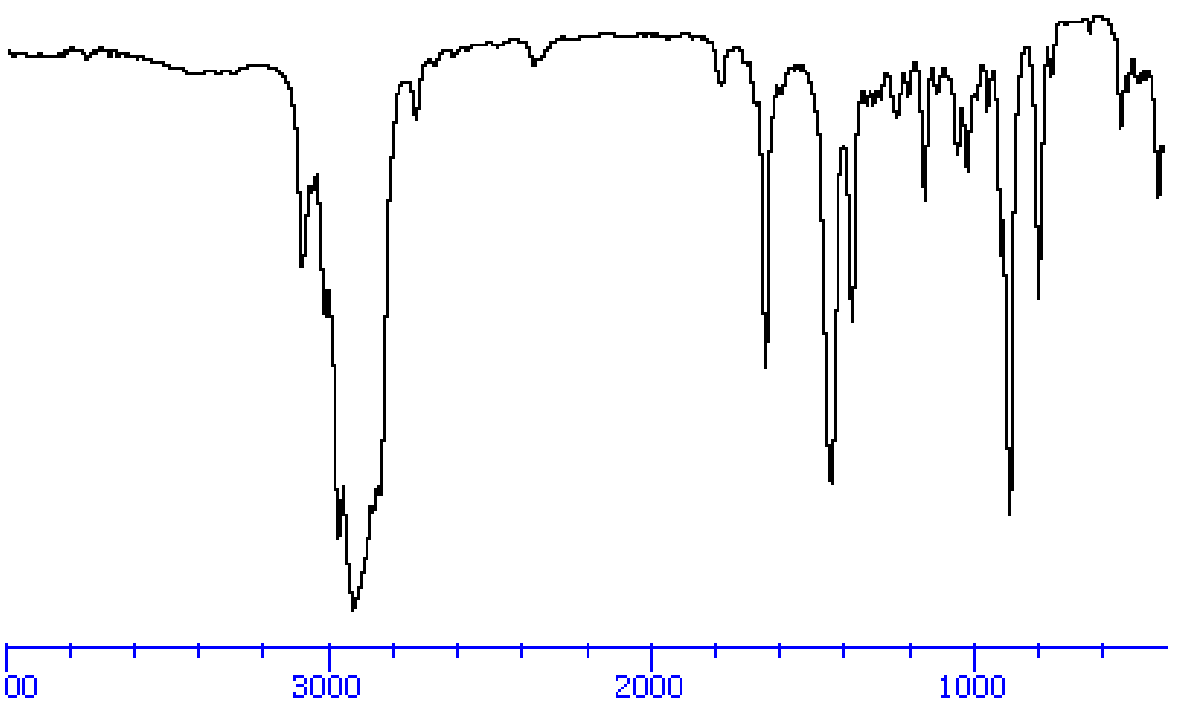

Figure 5. IR Diagram of d Limonene.

\section{Conclusion}

IR Spectroscopy is an extremely effective method for determining the presence or absence of a wide variety of functional groups in a molecule; IR spectra can be used to identify molecules by recording the spectrum for an unknown and comparing this to a library or data base of spectra of known compounds. Computerized spectra data bases and 
digitized spectra are used routinely in this way in research, medicine, criminology, and a number of other fields. The ingredients obtained from this study indicate that the essential oil of Lemon (Citrus limon L.) can be fully utilized for the manufacture of perfumery products, antimicrobial and antiseptic products.

\section{References}

[1] D. R. Caccioni, M. Guizzardi, D. M. Biondi, R. Agatino and G. Ruberto. Relationship between volatile components of citrus fruit essential oils and antimicrobial action on Penicillium digitatum and Penicillium italicum. Int. J. Food Microbiol. 43 (1998) 73-79.

[2] A. H. Rushdy Mohamed. Chemical and biological evaluation of deterpenated orange and mandarin oils. PhD Thesis, AlAzhar University. (2015) 3.

[3] K. Fisher and C. Phillips. Potential antimicrobial uses of essential oils in food: is citrus the answer? Trends Food. Sci. Tech. 19 (2008) 156.

[4] J. S. Baik, S. S. Kim, J. A. Lee, T. H. Oh, J. Y. Kim, N. H. Lee and C. G. Hyun. Chemical composition and biological activities of essential oils extracted from Korean endemic citrus species. J. J. Microbiol. Biotechn. 18 (2008) 74-79.

[5] D. C. Smith, S. Forland, E. Bachanos, M. Matejka and V. Barrett. Chemical Educator. Qualitative analysis of citrus fruits extracts by GC/MS: An undergraduate experiment. Chem. Educator. 6 (2001) 28-31.

[6] G. Flamini, M. Tebano and P. L. Cioni. Volatiles emission patterns of different plant organs and pollen of Citrus limon. Chim. Acta. 589 (2007) 120-124.
[7] K. P. Svoboda and R. I. Greenaway. Lemon scented plants. International Journal of Aromatherapy. 13 (2003) 23-32.

[8] The chemistry of essential oils, and their chemical components. http://essentialoils.co.za/components.htm. Accessed on: 27/02/2013 at $20 \mathrm{~h} 00$.

[9] M. Meskine and O. Ouardi. Analysis of the rovibrational frequencies of octahedral molecules. Revue Algérienne de Physique. 04 (2010) 62-68.

[10] A. Robert and A. Lobstein. Plantes aromatiques: épices, aromates, condiments et huiles essentielles. Éd. Tec \& doc, Paris. (2005) 522.

[11] D. Roux. Conseil en aromathérapie, 2 ème édition, proofficina (Rueil Malmaison), France. (2008) 187.

[12] R. Chiej. Green Guide, Medicinal plants, Edition Solar. (1982) 331.

[13] A. Basil and M. M. Jimenez-carmonna. Extraction of rosemary by super-heated water. Journal of food chemistry. 46 (1998) 5205-5209.

[14] H. Boughendjioua and S. Djeddi. Biological Activity of Lemon Essential Oil Used for Skin Care. European Journal of Scientific Research. (2014) 219-226.

[15] R. F. Dias. Spectroscopy: How do we know what organic molecules look like? Organic Chemistry (CHEM 311). (2005) 48-58.

[16] B. Elzey, V. Norman, J. Stephenson, D. Pollard, S. O. Fakayode. Purity Analysis of Adulterated Essential Oils by FT-IR Spectroscopy and Partial-Least-Squares Regression. Applications of Portable Raman Spectroscopy. (2016) 26-37. 